
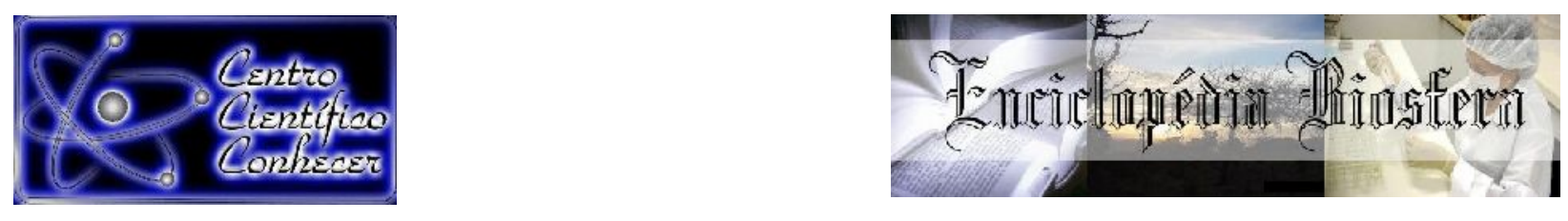

\title{
AÇÃO ANTIOXIDANTE DA CURCUMINA (CURCUMA LONGA L.) NA INJÚRIA DE ISQUEMIA E REPERFUSÃO TECIDUAL
}

Juliana Carvalho de Almeida Borges ${ }^{1}$, Klaus Casaro Saturnino ${ }^{2}$, Vanessa de Sousa Cruz ${ }^{3}$, Eugênio Gonçalves de Araújo ${ }^{4}$

1 Doutoranda do Programa de Pós-Graduação em Ciência Animal da Escola de Veterinária

e Zootecnia da Universidade Federal de Goiás (juliana_vet_ufg@hotmail.com).Goiás-Brasil

2 Professor Doutor do Setor de Patologia Veterinária da Universidade Federal de Goiás Regional Jataí

3 Pós doutoranda, Laboratório Multiusuário de Cultivo Celular da Universidade Federal de Goiás

4 Professor Doutor do Setor de Patologia Veterinária da Universidade Federal de Goiás

\section{Recebido em: 06/04/2019 - Aprovado em: 10/06/2019 - Publicado em: 30/06/2019 DOI: 10.18677/EnciBio_2019A4}

\begin{abstract}
RESUMO
Doenças do sistema circulatório estão entre as principais causas de morbidade e mortalidade em humanos, suas repercussões clínicas geram co-morbidades que elevam negativamente os indicadores de saúde populacional, além de dispendiosos gastos públicos. A maioria dessas doenças ocorre por mecanismos isquêmicos. Lesão tecidual por isquemia é nociva ao organismo, entretanto o restabelecimento do fluxo sanguíneo pode levar a uma série de complicações, que exacerbam ainda mais os danos teciduais, devido à produção de EROs (espécies reativas do oxigênio). Antioxidantes são substâncias endógenas ou exógenas, que protegem as células contra o efeito das EROs. Entre os antioxidantes exógenos presentes nos vegetais, os mais ativos e encontrados são os flavonóides. A cúrcuma (Curcuma Longa L.) é uma planta originária da Índia e do sudeste da Ásia, derivada dos flavonóides. A parte mais utilizada é a raiz, que apresenta pigmento amarelo intenso, conhecido popularmente como açafrão. Seu principal pigmento curcuminóide é a curcumina. No Brasil, a cúrcuma é mais produzida e consumida no Centro-Oeste e em Minas Gerais. Em Goiás, a região de Mara Rosa é responsável pela produção de 800 a mil toneladas de açafrão, por ano. Muitos produtores são agricultores familiares ou arrendatários e essa cadeia produtiva gera emprego e renda para a população local. O teor de curcumina encontrado no açafrão de Mara Rosa, é de $6,8 \%$, valor bastante significativo em comparação ao cultivares da Índia, maior fornecedor mundial. Esta revisão de literatura compila algumas comprovações científicas quanto à atividade antioxidante da cúrcuma, em tecidos submetidos à isquemia e reperfusão.
\end{abstract}

PALAVRAS-CHAVE: curcumina, isquemia, Mara Rosa. 


\title{
ANTIOXIDANT ACTION OF CURCUMINE (CURCUMA LONGA L.) IN THE TISSUE ISCHEMIA-REPERFUSION INJURY
}

\begin{abstract}
Diseases of the circulatory system are among the leading causes of morbidity and mortality in humans, and its clinical repercussions generate co-morbidities that elevate negatively the indicators of population health, besides costly public expenses. Most of these diseases occurs by ischemic mechanisms. Injury by ischemia is harmful, however the restoration of blood flow can lead to a series of complications, which further exacerbate the damage to the tissue, due to the production of ROS (reactive oxygen species). Antioxidants are substances that protect cells against the effect of ROS. Among the antioxidants exogenous present in the vegetables, the most actives and found are the flavanoids. Turmeric (Curcuma Longa L.) is a native plant from India and southeast Asia, which belongs to the subclass of curcuminoids, derived from flavonoids. The used part of turmeric is the root, that presents an intense yellow pigment, popularly known as saffron. Its main curcuminoid pigment is the curcumin. In Brazil, turmeric is more produced and consumed in the Center-West and Minas Gerais. In Goiás, the Mara Rosa region is responsible for the production of 800 to 1000 tons of saffron, per year. Many producers are family farmers or tenants, this production chain generates employment and income for the local population. The curcumin content found in the saffron, produced in Mara Rosa, is $6,8 \%$, quite significant value in comparison to the cultivars from India, the world's largest supplier. This literature review compiles some scientific evidence on the antioxidant activity of turmeric in tissues submitted to ischemia and reperfusion.
\end{abstract}

KEYWORDS: curcumin, ischemia, Mara Rosa.

\section{INTRODUÇÃO}

A cúrcuma (Curcuma Longa L.) é uma planta perene, originária da Índia e do sudeste da Ásia. Curcumina é o seu principal componente, pigmento de coloração amarelo-alaranjado que caracteriza o popular açafrão. Utilizada como condimento ou corante alimentar, a cúrcuma apresenta também atividades farmacológicas relatadas desde a antiguidade (ALONSO, 2007).

No Brasil, o sistema público de saúde passou a utilizar plantas com potencial medicinal e, em 2009, o Ministério da Saúde publicou a Relação Nacional de Plantas Medicinais de Interesse ao Sistema Único de Saúde (RENISUS), em que se encontra listada a cúrcuma. Considerando essa perspectiva, constantes pesquisas etnofarmacológicas são desenvolvidas no país e em outras nações para incorporação de bioativos com ação farmacológica eficaz, comprovados cientificamente, na indústria farmacêutica. Essa prática é bastante visada pelas entidades governamentais, que subsidiam onerosos custos medicamentosos às suas populações e buscam alternativas mais vantajosas, economicamente (BRASIL, 2009).

Doenças do sistema circulatório estão entre as principais causas de morbidade e mortalidade em humanos, suas repercussões clínicas geram comorbidades que elevam negativamente os indicadores de saúde populacional, além de dispendiosos gastos públicos (BRASIL, 2014). A maioria dessas doenças vasculares ocorrem por mecanismos de isquemia e reperfusão $(\mathrm{l} / \mathrm{R})$, em que o tecido é lesionado pela produção em excesso de espécies reativas do oxigênio (ERO). 
Esse acúmulo é conhecido por estresse oxidativo, situação em que a produção de ERO está superior a capacidade antioxidante do organismo. Uma quantidade considerável de estudos, em humanos e animais, demonstra os efeitos benéficos da ação de antioxidantes exógenos, provenientes da alimentação, nos tecidos acometidos por I/R (BIANCHI; ANTUNES, 1999).

A cúrcuma apresenta efeito pleitotrópico, incluindo ações antioxidante, antifúngica, anti-inflamatória, antibacteriana, antitumoral, antiviral, cicatrizante, hipoglicemiante, neuroprotetora, antiparasitária e imunomoduladora. Devido a isso, o uso da cúrcuma como suplemento já foi aprovado em diversos países, com aceitação e consumo amplo pela população (MARCHI et al., 2016).

Diante do exposto, objetivou-se com esta revisão de literatura apresentar o que se tem descoberto e comprovado cientificamente quanto à atividade antioxidante da cúrcuma, demonstrar sua importância terapêutica contra doenças e estimular o uso dos recursos naturais disponíveis e, principalmente, de custo acessível tanto na medicina quanto na veterinária.

\section{CARACTERIZAÇÃO DA CÚRCUMA (CURCUMA LONGA L.)}

A Curcuma Longa $\mathrm{L}$. é uma planta perene de longas ramificações laterais, pertencente à família das Zingiberaceae, sob o gênero Curcuma L., originária da India e de ocorrência em regiões tropicais e subtropicais (PRABHAKARAN, 2013). No Brasil, é mais produzida e consumida no Centro-Oeste e em Minas Gerais. É conhecida popularmente por açafrão, açafroa, açafrão-da-terra, açafrão-da-índia, falso-açafrão, gengibre-dourado, gengibre-amarelo, batatinha-amarela, cúrcuma, mangarataia, turmérico ou turmerique (MARCHI et al., 2016). A parte mais comumente utilizada da cúrcuma é a raiz ou rizoma. Sua colheita pode ser feita logo após a floração, período em que a planta começa a secar e seus rizomas apresentam pigmentos amarelos intensos. Estes são amplamente empregados como corante, tempero ou condimento alimentar, na forma de pó seco, posterior ao seu processamento (Figura 1) (BANIK et al., 2017).

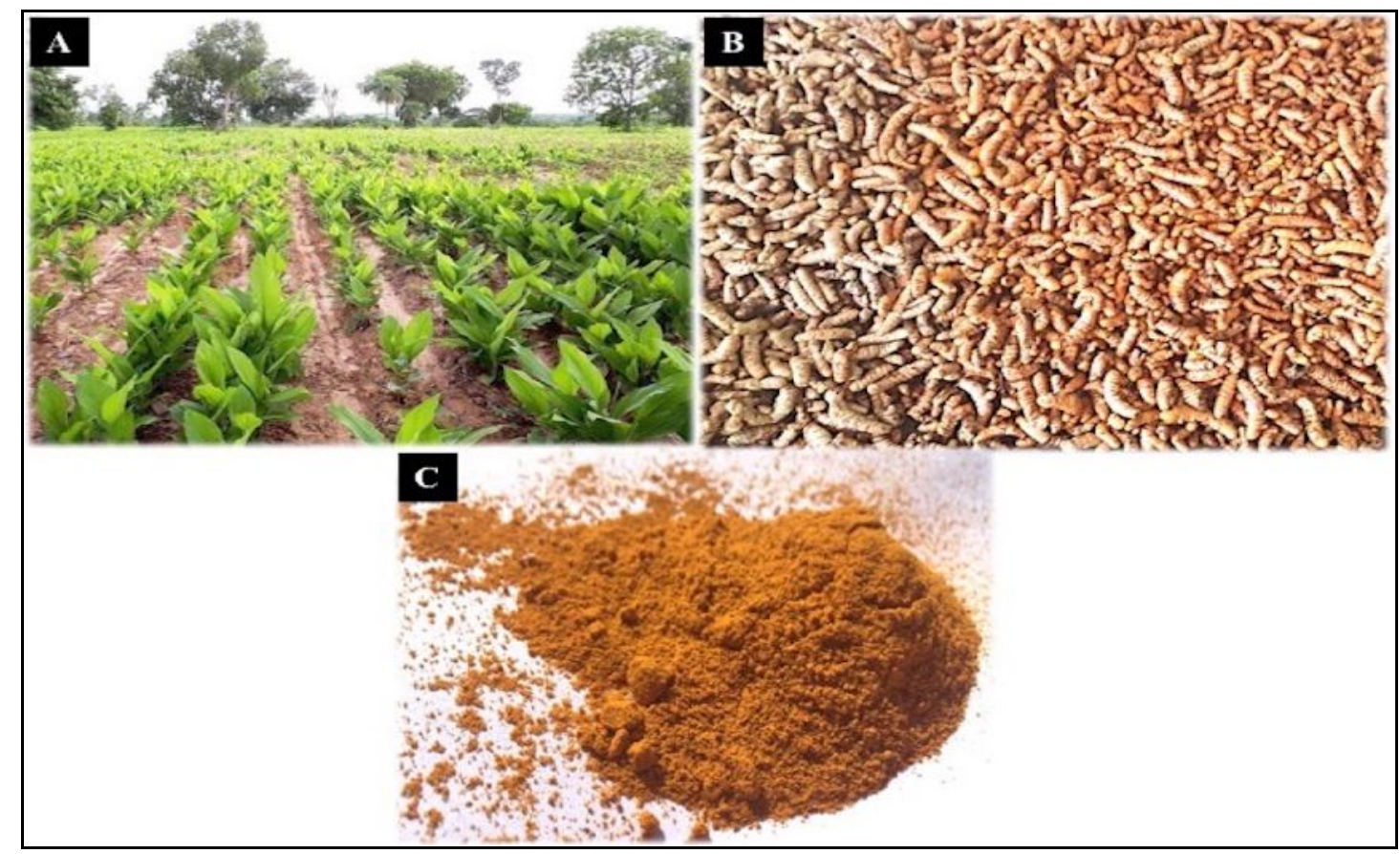


FIGURA 1 - Imagem da plantação de Curcuma longa L. em Mara Rosa - GO (A). Rizoma íntegro (B). Rizoma após o processamento a pó (C).

FONTE: Arquivo pessoal,( 2018).

Em Goiás, a região que abrange os municípios de Mara Rosa, Amaralina, Formoso e Estrela do Norte é responsável pela produção de 800 a mil toneladas de açafrão desidratado por ano. De acordo com a Cooperativa dos Produtores de Açafrão de Mara Rosa, a região movimenta anualmente mais de $R \$ 1$ milhão e como a maioria dos produtores é constituída por arrendatários e agricultores familiares, a cadeia produtiva gera emprego e renda para a população local. O solo e o clima de Goiás são propícios para o cultivo da cúrcuma, e a região norte recebeu o selo ou registro de Identidade Geográfica do Estado de Goiás para a produção de açafrão, pelo Instituto Nacional de Propriedade Industrial, o que agregou valor ao produto (DANTAS, 2016).

Outro diferencial do produto da região de Mara Rosa é o teor de curcumina, responsável pela pigmentação dos rizomas. A quantidade média encontrada desse composto foi de $6,8 \%$, valor bastante significativo em comparação aos maiores teores de curcumina relatados na literatura, que se encontram em cultivares na Índia, o maior fornecedor mundial (SILVA, 2009).

\section{COMPOSIÇÃO FENÓLICA DA CÚRCUMA (CURCUMA LONGA L.)}

Os compostos fenólicos têm sido objetos de investigação desde a descoberta de suas ações antioxidantes. Os organismos vivos estão, constantemente, submetidos à ação oxidativa do oxigênio. O processo respiratório e as diversas reações biológicas das células aeróbicas como apoptose, crescimento celular, inflamação e secreção de substâncias levam à formação de moléculas intermediárias, denominadas radicais livres ou ERO (PONNUCHAMY; KHALIL, 2009).

Estresse oxidativo corresponde a um desequilíbrio entre a produção das espécies reativas e a capacidade antioxidante do organismo. Tal disparidade propicia o surgimento de diversas doenças crônicas não-transmissíveis, incluindo hipertensão, aterosclerose, diabetes, doenças cardiovasculares, acidente vascular encefálico (PANDEY; RISVI, 2009).

O organismo apresenta mecanismos endógenos e exógenos de defesa contra o estresse oxidativo, que atuam prevenindo, reparando ou inativando as reações moleculares dos elétrons desemparelhados. O sistema enzimático, constituído pelas enzimas: catalase, metionina-redutase, superóxido-dismutase e sistemas tioredox e glutationa, é um dos integrantes do grupo endógeno de inibição dos efeitos da oxidação. O sistema antioxidante não-enzimático, também faz parte do grupo endógeno e compõe-se de melatonina, bilirrubina, proteínas de ligação de metal, ácido úrico, poliaminas, entre outros (MIROŃCZUK-CHODAKOWSKA et al., 2018).

As pesquisas têm demonstrado uma estreita correlação entre o consumo de frutas, legumes, castanhas, cereais, certas bebidas, e a redução no risco de doenças crônicas não-transmissíveis. Isso ocorre devido à presença de compostos antioxidantes nesses alimentos, o que caracteriza um mecanismo exógeno de defesa do organismo. Compostos fenólicos são estruturas químicas que contêm um grupo hidroxila $\left(\mathrm{OH}^{-}\right)$ligado diretamente ao átomo de carbono, em um anel benzênico. Os fenóis podem ser encontrados sob forma de polifenóis (flavanóides) ou fenóis simples (não-flavanóides). Os flavanóides são os mais frequentemente 
encontrados em frutas e vegetais e apresentam estrutura química de 15 átomos de carbono, arranjado em três anéis de benzeno (ZUJKO et al., 2012).

A cúrcuma pertence à subclasse de curcuminóides, derivada dos flavanóides. Os principais pigmentos curcuminóides presentes no rizoma da planta são a curcumina (77\%), a desmetoxicurcumina (17\%) e a bisdemetozicurcumina (3\%), análogos estruturalmente, diferenciando-se apenas pela quantidade de grupos metoxila $\left(\mathrm{OCH}_{3}\right)$ em sua estrutura química. A curcumina é o pigmento mais ativo e também é o que se encontra em maior concentração na cúrcuma. Sob a fórmula molecular $\mathrm{C}_{21} \mathrm{H}_{20} \mathrm{O}_{6}$, a curcumina pode apresentar-se por meio de duas formas tautoméricas, a forma cetônica e a forma enólica, das quais a última é a mais estável tanto em fase sólida quanto em solução. Em pH básico, a forma enólica está presente em maior quantidade, atuando principalmente como um doador de elétrons, o que caracteriza sua atividade antioxidativa (ANJOMSHOA et al., 2016).

A maioria das pesquisas nas quais se avalia a atividade antioxidante da Curcuma longa L. é realizada por meio de tratamentos das amostras com extrato aquoso ou etanólico da parte específica da planta. As formas mais comuns de obtenção da fração ativa são por maceração estática e dinâmica, decocção, infusão, percolação, extrator de Soxhlet, refluxo, extração por turbólise, ultrassom e hidrodestilação para os óleos essenciais. As técnicas de identificação dos compostos fenólicos envolvem cromatografia líquida de alta eficiência, cromatografia gasosa, cromatografia de permeação em gel e espectroscopia na região do infravermelho (FTIR) (BRASIL, 2015).

A biotransformação ou metabolismo da cúrcuma depende diretamente de sua forma de administração. Estudos demonstraram que a curcumina apresenta baixa biodisponibilidade, o que se torna limitante sua utilização clínica. Essa questão pode estar relacionada à reduzida solubilidade em água devido à sua característica lipofílica, tornando-a pouco absorvível, além da rapidez na metabolização e eliminação do organismo. O uso da via oral para a liberação de substâncias farmacologicamente ativas é a mais comum, apesar do fato de elas serem altamente degradáveis pelo trato gastrointestinal. A curcumina sofre metabolismo hepático antes de alcançar a circulação sistêmica, além de metabolismo intestinal, o que reduz drasticamente sua fração utilizável nos órgãos-alvo (OZAWA, et al., 2017).

Compostos fenólicos, em geral, são metabolizados no fígado por conjugação a ácido glicurônico ou ácido sulfúrico. Após atingir a corrente sanguínea, a molécula de curcumina praticamente não é mais evidenciada em sua fração livre no plasma; em decorrência disto, a elucidação de seus mecanismos de metabolização e biodisponibilidade tornam-se mais laboriosos. Com a finalidade de aumentar a biodisponibilidade da curcumina, muitos estudos têm utilizado diversas estratégias para incorporar maior resistência ao composto, dentre elas pode-se citar associação à piperina, fracionamento a nanopartículas, lipossomas, micelas ou complexos fosfolipídicos e curcumina- $\beta-D$-glucuronida (curcumina monoglucuronida) (OZAWA, et al., 2017).

Pesquisadores apontaram que após a administração de curcumina em ratos pela via oral, a molécula era rapidamente biotransformada em suas formas conjugadas; no entanto, a forma ativa do composto no sangue era extremamente baixa. A curcumina- $\beta$-D-glucuronida (Figura 2 ) é o principal metabólito da curcumina, porém após a sua administração pela via intravenosa nos animais, verificou-se que ela foi convertida em curcumina na forma livre, ou seja, forma ativa do composto. 
Entretanto, com a administração oral, esses mesmos níveis não puderam ser alcançados (SHOJl et al., 2014).

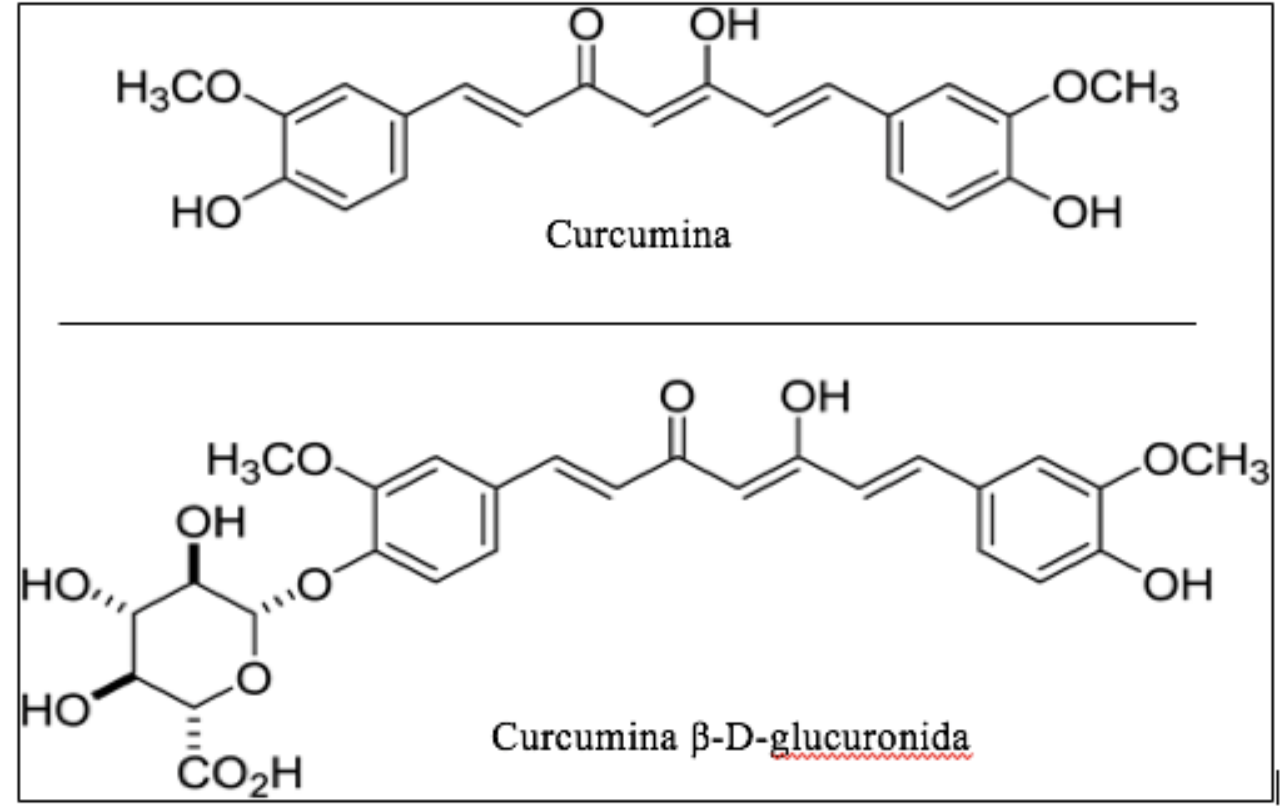

FIGURA 2 - Estrutura química da curcumina e de seu metabólito principal, curcumina $\beta$-D-glucuronida (curcumina monoglucuronida).

FONTE: OZAWA et al.,(2017).

Esses resultados são sugestivos que a curcumina monoglucuronida pode ser aplicável como um pró-fármaco, da curcumina livre, controlado pelo metabolismo. Isso viabiliza a utilização terapêutica do composto, principalmente em doenças crônicas não-transmissíveis, que tem como principal barreira sua disponibilidade no organismo ${ }^{25,27}$.

\section{MECANISMO DE ISQUEMIA E REPERFUSÃO TECIDUAL}

As referências estatísticas sobre as causas de mortalidade em um país, constituem a forma mais usual para se ter conhecimento sobre o nível de saúde de seus habitantes e planejar ações de prevenção e promoção da saúde. Doenças do sistema circulatório estão entre as principais causas de morbidade e mortalidade na população de vários países, incluindo o Brasil (BRASIL, 2014). Muitas delas cursam com o processo de isquemia e reperfusão e como consequência geram ERO em quantidade superior à capacidade antioxidante do sistema. Diversos estudos na área preventiva têm sido realizados para amenizar essa realidade, prevalecendo o uso dos anioxidantes exógenos (GAUl et al., 2014).

É notório que a lesão tecidual por isquemia é nociva ao organismo; entretanto, o restabelecimento do fluxo sanguíneo pode levar a uma série de complicações, que exacerbam ainda mais os danos teciduais. Isquemia é uma condição em que o fluxo sanguíneo, rico em oxigênio, é restringido ou interrompido a determinado órgão ou tecido, prejudicando sua oxigenação celular. No decorrer de uma isquemia, o metabolismo celular aeróbico é suprimido, e ocorre somente o metabolismo anaeróbico; em consequência, as mitocôndrias, organelas 
responsáveis por converter o oxigênio $\left(\mathrm{O}_{2}\right)$ em energia para as células, sofrem alterações bioquímicas e morfológicas que podem evoluir para morte celular e disfunção do órgão afetado (KALOGERIS et al., 2016).

A supressão de $\mathrm{O}_{2}$ induz à anaerobiose celular, em que há acentuada queda de $\mathrm{pH}$ e ativação de proteases intracelulares. Nas membranas basolaterais das células há canais denominados trocadores $\mathrm{Na}^{+} / \mathrm{H}^{+}$que visam reestabelecer o $\mathrm{pH}$ alterado, em consequência do acúmulo de $\mathrm{H}^{+}$. Eles excretam o excesso deste íon, mas em consequência produzem um elevado efluxo de $\mathrm{Na}^{+}$. O acúmulo de $\mathrm{Na}^{+}$ também ocorre por diminuição da atividade da bomba de $\mathrm{Na}^{+} / \mathrm{K}^{+}$, com subsequente aumento do influxo de água, induzindo ao edema citotóxico, conhecido por degeneração hidrópica (KUMAR et al., 2013).

A depleção de oxigênio inibe a fosforilação oxidativa e, portanto, diminui a produção de adenosina trifosfato (ATP) pela mitocôndria. A célula é incapaz de manter sua função e o gradiente transmembrana desaparece, induzindo o aumento do influxo de cálcio $\left(\mathrm{Ca}^{+2}\right)$ que culmina em excesso intracelular. Uma maior concentração, desse íon, ativa enzimas destrutivas como as fosfolipases, as proteases e as endonucleases, que causam desarranjo das membranas das organelas e dos componentes do citoesqueleto. O dano mitocondrial ocorre pela degradação dos fosfolipídeos e por produtos da degradação lipídica. Essas mudanças são acompanhadas pela abertura do poro de transição de permeabilidade mitocondrial (TPM), que dissipa o potencial da membrana e prejudica ainda mais a produção de ATP. Entretanto essas reações podem ainda ser reversíveis e a célula pode apresentar uma resposta adaptativa, porém se a permanência do fator desencadeante prevalecer, haverá morte celular (HAUSENLOY; YELLON, 2013).

Durante a respiração aeróbica, uma quantidade bastante reduzida de ERO é produzida por intermédio do metabolismo do oxigênio. Essa produção é usualmente neutralizada pelos sistemas antioxidantes enzimáticos, presentes nas mitocôndrias, como superóxido dismutase (SOD) e glutationa peroxidase (GPX). O estresse oxidativo se estabelece durante o período de reperfusão, em que a produção de ERO supera a capacidade eliminatória do sistema (GHOLAMPOUR; SADDID, 2018).

Reperfusão é o termo utilizado para definir o restabelecimento do fluxo sanguíneo e, portanto, do $\mathrm{O}_{2}$, ao tecido que se encontrava em isquemia. No entanto, a reoxigenação agrava ainda mais a lesão isquêmica. Sabe-se que a principal causa da lesão de reperfusão é a abertura permanente, dos poros de TPM. A acidez provocada pela queda de $\mathrm{pH}$ durante a isquemia evita a fissura desses poros; todavia, durante a reperfusão, há uma restauração do $\mathrm{pH}$ fisiológico, que estimula sua abertura. Como consequência, tem-se o rompimento da membrana mitocondrial, que libera componentes pró-apoptóticos como o fator indutor da apoptose e o citocromo- $C$, e se tem a entrada acentuada de prótons, alterando o potencial de membrana mitocondrial, que compromete a síntese de ATP (VERCESI et al., 2018).

Os mecanismos implicados no retorno de oxigênio molecular ao tecido são complexos e envolvem sobrecarga de cálcio, permanência de abertura de poros TPM, disfunção endotelial, produção de citocinas, respostas inflamatórias além de geração de ERO (HAUSENLOY; YELLON, 2013).

No período de isquemia, o ATP é catabolizado gradativamente à ADP e $\mathrm{AMP}$, e, posteriormente, à adenosina, inosina e hipoxantina. Paralelamente, uma 
protease citosólica ativada pelo $\mathrm{Ca}^{2+}$, acumulado no citosol, converte xantina desidrogenase em xantina oxidase (OX). Com a reperfusão, a hipoxantina pode ser oxidada à xantina, pela OX, que em seguida é convertida em ácido úrico. Durante o processamento dessas reações químicas, ERO são geradas como subprodutos, entre elas o ânion superóxido $\left(\mathrm{O}_{2}{ }^{-}\right)$, peróxido de hidrogênio $\left(\mathrm{H}_{2} \mathrm{O}_{2}\right)$ e radicais hidroxila $\left(\mathrm{OH}^{-}\right)$, esquematizado na Figura 3 (SILVA et al., 2002).

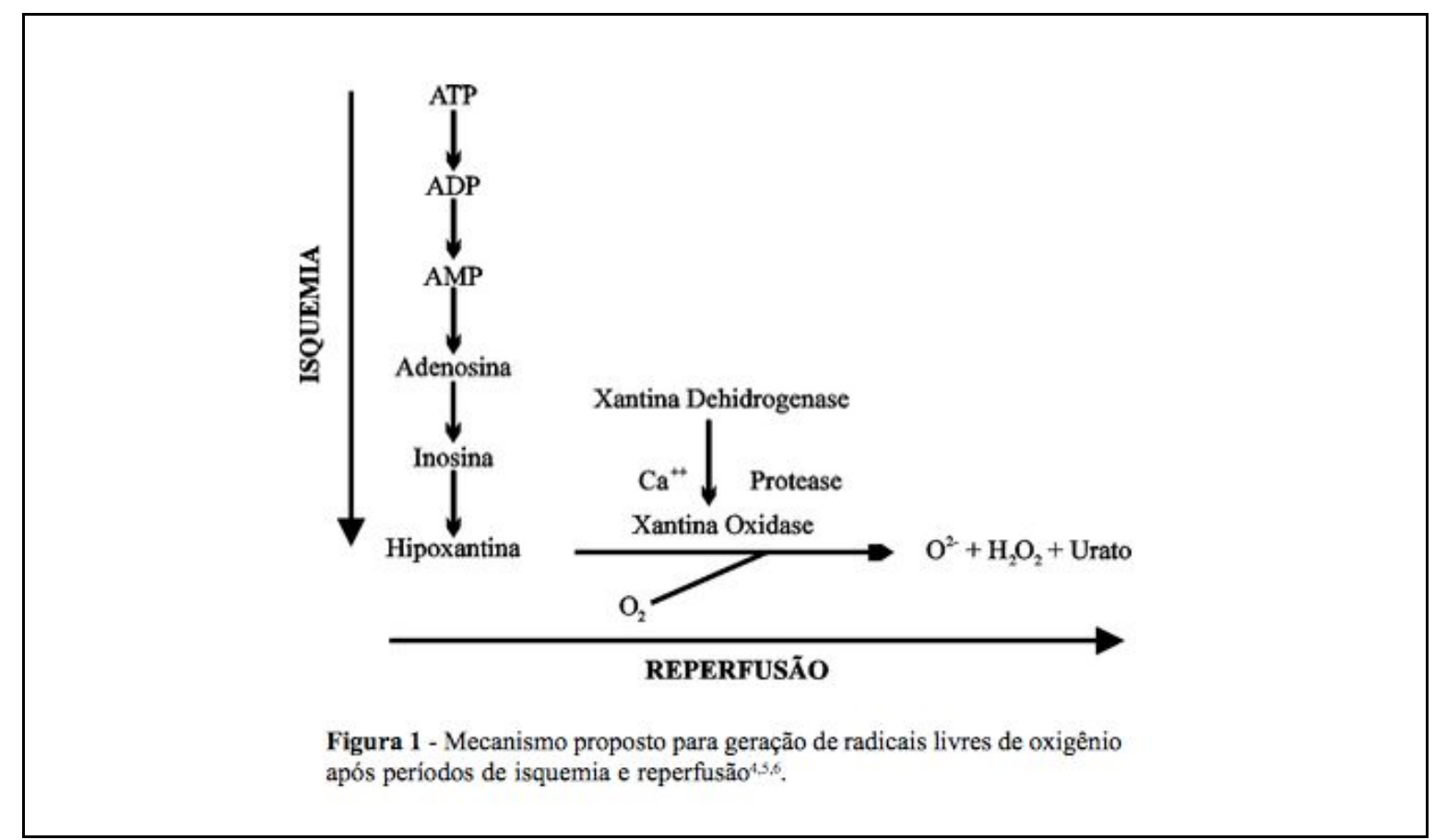

FIGURA 3 - Esquema das reações químicas geradas durante a isquemia e reperfusão tecidual, com produção de espécies reativas de oxigênio $\left(\mathrm{O}_{2}\right)$. Durante o período de isquemia, o ATP é catabolizado à hipoxantina, mas com o retorno do $\mathrm{O}_{2}$ da reperfusão ocorrem oxidações que resultam em liberação de espécies reativas de $\mathrm{O}_{2}$.

FONTE: SILVA et al., (2002).

Muito se tem relatado no meio científico e divulgado para a população em geral sobre os efeitos benéficos dos antioxidantes da dieta, na prevenção da formação de ERO e também na reparação de seus efeitos deletérios nas células. A curcumina tem ganhado espaço nesse contexto, devido à sua estrutura molecular funcional, capaz de provocar reações químicas biologicamente importantes como: doação de prótons hidrogênio (oxidação), hidrólise, degradação e outras reações enzimáticas (PRIYADARSINI, 2014).

As moléculas antioxidantes atuam nas reações de abstração de hidrogênio e também de transferência de elétrons. Os três sítios ativos da curcumina podem sofrer oxidação por transferência de elétrons e abstração de hidrogênio, mas algumas pesquisas sugerem que o hidrogênio mais facilmente extraível, da curcumina, é o pertencente ao grupo fenólico $(\mathrm{OH})$. A reação química resulta na formação do radical fenoxil, que é bem mais estável e menos reativo (Figura 4) (PRIYADARSINI, 2014). 


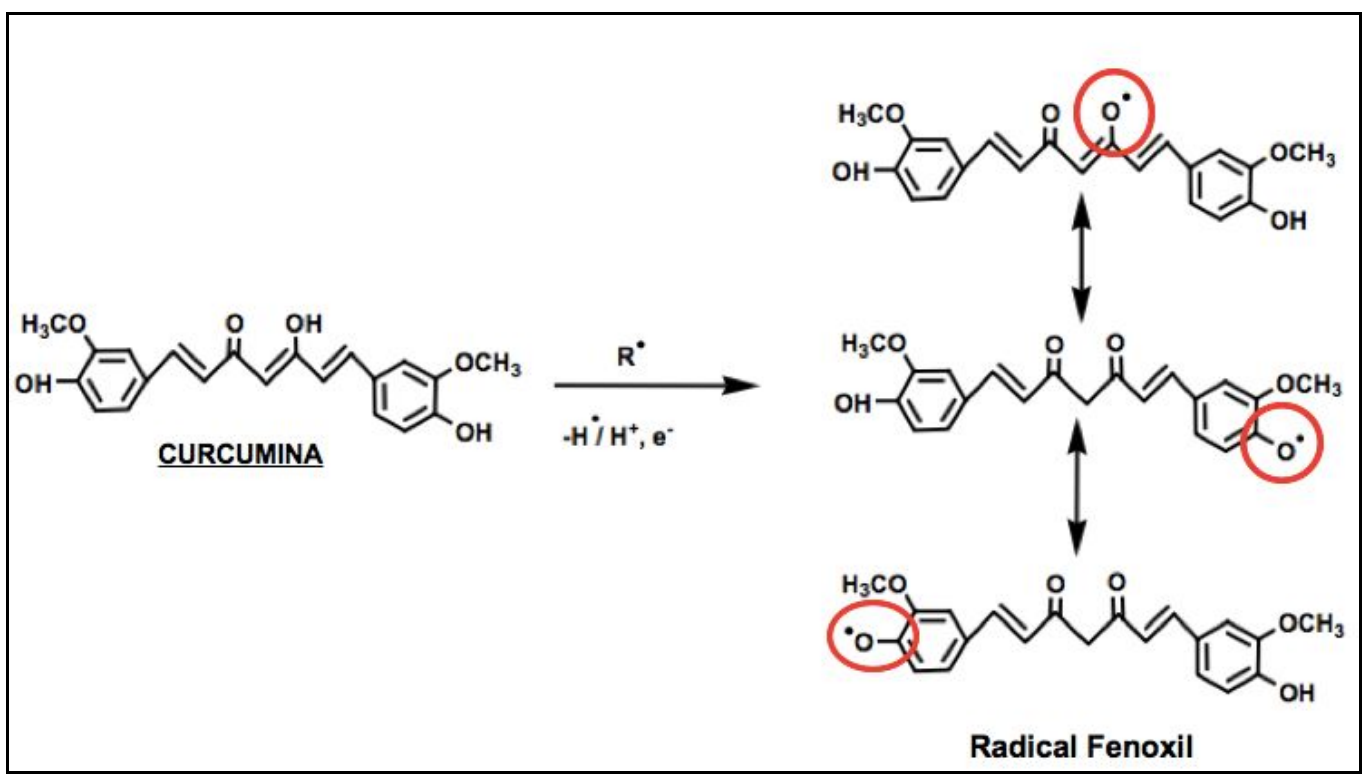

FIGURA 4 - Esquema dos sítios de ação da curcumina para as espécies reativas do oxigênio $\left(\mathrm{O}_{2}-\right.$ círculos $)$.

FONTE: PRIYADARSINI, (2014).

\section{EFEITOS TERAPÊUTICOS DA CURCUMINA NA ISQUEMIA E REPERFUSÃO}

Todos os anos, a Associação Americana de Cardiologia (American Heart Association) publica dados epidemiológicos relacionados a doenças cardiovasculares, acidentes vasculares encefálicos e doenças metabólicas, coletados com o auxílio de outras instituições de saúde, públicas e privadas. No entanto, apesar de todo conhecimento que se tem e as constantes pesquisas na área de prevenção e atenuação, os acometimentos cardíacos continuam, invariavelmente, como primeira causa de mortalidade, em todo o mundo (MOZAFFARIAN et al., 2016).

A curcumina tem sido importante alvo de pesquisas nas doenças cardíacas. Seus efeitos protetores, relatados desde a antiguidade, são convidativos aos pesquisadores para investigarem suas ações em tecidos cardíacos. Cientistas chineses investigaram a atividade protetora da molécula perante a injúria de $\mathrm{I} / \mathrm{R}$ miocárdica, tanto in vivo quanto in vitro, e relataram efeito cardioprotetor. $\mathrm{O}$ uso da curcumina anteriormente à $\mathrm{I} / \mathrm{R}$ amenizou os efeitos deletérios da alteração circulatória, o que foi confirmado por meio do registro de uma área de infarto significativamente menor, além de menor índice apoptótico. A proteína inibidora de apoptose, Bcl-2, apresentou maior expressão nos tecidos, ao contrário da proteína Bax, pró-apoptótica, que se manteve em nível reduzido. Preservou-se a função mitocondrial, principalmente por conseguir manter o potencial redox com ativação da enzima antioxidante endógena superóxido dismutase (SOD) e diminuição de formações de $\mathrm{H}_{2} \mathrm{O}_{2}$ e malondialdeído (MDA). Acreditou-se que esses fatores derivaram primariamente da ativação da proteína de sobrevivência celular - SIRT1 (YANG et al., 2013).

Em semelhante pesquisa, ratos foram submetidos à isquemia por oclusão da artéria coronariana, seguida de três horas de reperfusão. Os animais foram previamente suplementados com curcumina oral via gavagem por 20 dias e apresentaram os seguintes resultados, conforme níveis crescentes de curcumina 
utilizada: (1) melhor desempenho das funções sistólica e diastólica pós-isquêmicas, devido evolução na contratilidade cardíaca e diminuição da demanda de oxigênio; (2) diminuição dos níveis de MDA e aumento de enzimas antioxidantes (SOD, catalase, glutationa redutase, glutarredoxina); (3) efeito antiapoptótico, pela redução da expressão miocárdica de Bax e Caspase-3 (via final apoptótica) e pela elevação do nível de Bcl-2. Ademais, o efeito cardioprotetor da curcumina se confirmou por diminuição sérica das enzimas indicadoras de dano miocárdico LDH, CK-MB e aumento de $\mathrm{Ca}^{2+-}$ ATPase e $\mathrm{Na}^{+}-\mathrm{K}^{+}$-ATPase, com redução da área de infarto (LIU et al., 2017).

O acidente vascular encefálico (AVE) de origem isquêmica é a segunda causa mais comum de mortalidade e principal causa de incapacidade entre a população adulta acometida. Restaurar a circulação sanguínea para a área isquêmica do cérebro é atualmente a única terapia aprovada pela Food and Drug Administration dos Estados Unidos. No entanto, essa reperfusão estimula células inflamatórias e agentes relacionados à apoptose, que alastram a lesão encefálica inicial. Por conseguinte, a utilização de compostos que possam amenizar a ação das células inflamatórias pode ser benéfica; nesse contexto, a curcumina apresenta propriedades que justificam seu uso. Ratos submetidos à oclusão da porção inicial da artéria cerebral média por uma hora e submetidos ao tratamento com curcumina intraperitoneal, pré-reperfusão, apresentaram diminuição da expressão das citocinas pró inflamatórias ICAM-1 e NF-kB, redução na marcação de metaloproteinase MMP9 (enzima envolvida na degradação da barreira hematoencefálica) e redução de caspases-3. A porcentagem da área infartada encefálica, foi significativamente menor, quando comparado ao grupo controle (LI et al., 2017). Esses resultados corroboraram com outra pesquisa, em que também se induziu $\mathrm{I} / \mathrm{R}$ encefálica em ratos, porém da porção distal da artéria cerebral média; foram encontraram significativas reduções tanto nas áreas de infarto, quanto na expressão de moléculas da inflamação. Dessa forma, a curcumina comprovou-se efetiva na atenuação da injúria de I/R em AVE (LIU et al., 2017). A Figura 5 demonstra o efeito protetor observado nos encéfalos, nos dois trabalhos analisados.

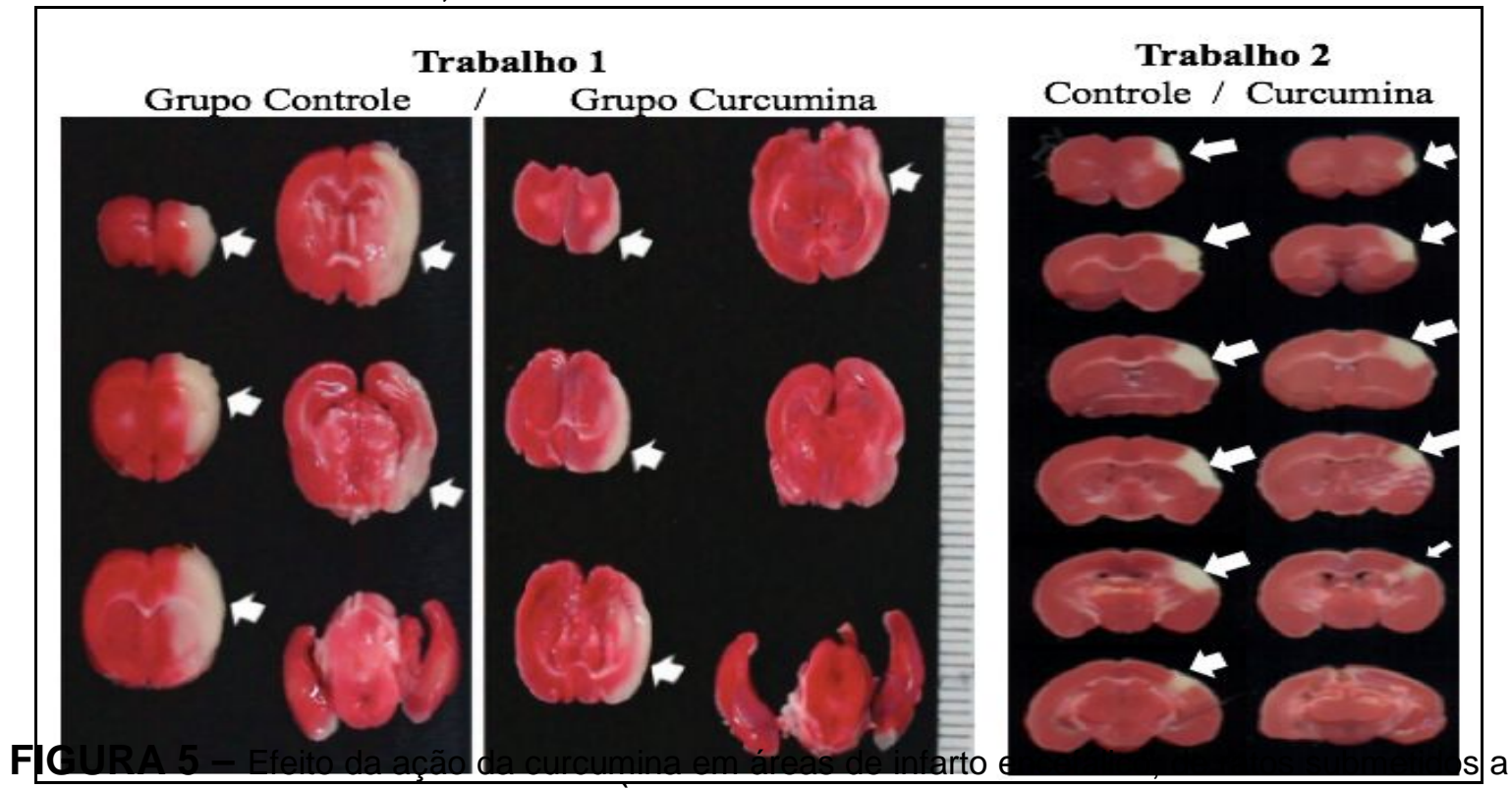

isquemia e reperfusão. À coloração de TTC (2,3,5- cloreto de trifeniltetrazólio), as áreas lesionadas surgiram como regiões brancas (setas). As amostras dos grupos 
que utilizaram curcumina apresentaram lesões menores em comparação aos grupos controle, tanto no trabalho 1 (esquerda) quanto no 2 (direita).

Fonte: LI et al., (2017) (Trabalho 1); LIU et al., (2017) (Trabalho 2).

A $1 / R$ renal também é bastante relatada na literatura. As causas mais comuns da redução do fluxo sanguíneo renal incluem nefrectomia parcial, transplante renal, hipovolemia, hipotensão, hipertensão, angioplastia da artéria renal e cirurgia de aneurisma da aorta. Resultados do uso da curcumina em modelos de I/R renal, por oclusão uni ou bilateral de artérias e veias renais de ratos, demonstraram a eficácia da molécula devido, principalmente, à sua dupla atividade antioxidativa, ao eliminar ERO diretamente e induzir indiretamente enzimas antioxidantes. Jacob et al. (2013) utilizaram a curcumina pela via oral em ratos com resposta imune deficiente e glomerulonefrite e observaram a redução da glomeruloesclerose e a melhora da função renal, associada à diminuição de marcadores inflamatórios (TGF- $\beta$ e MCP-1) e proteínas da matriz (fibronectina, laminina e colágeno).

Pesquisas in vitro também demonstraram o efeito protetor da curcumina. Células renais humanas (HK-2) foram submetidas à $\mathrm{I} / \mathrm{R}$ e como resposta, obteve-se diminuição de proliferações celulares, redução de células apoptóticas e por meio de coloração fluorescente avaliaram-se os níveis de produção de ERO. Uma menor intensidade de cor nas células tratadas com curcumina foi visualizada, o que sugere inibição da resposta ao estresse oxidativo. Verificaram-se também níveis de MDA diminuídos e da enzima antioxidativa, SOD, aumentados (XU et al., 2016).

Estudos em ratos nefrectomizados acometidos por proteinúria, hipertensão, arteriolopatia, inflamação intersticial tubular e alterações hemodinâmicas nos néfrons apresentaram redução tanto das alterações sistêmicas como glomerulares. Além da atividade aumentada das enzimas antioxidantes e diminuição no estresse oxidativo, o uso preventivo da curcumina reduziu a proteinúria, pressão arterial sistólica, glomeruloesclerose, danos intersticiais tubulares e marcadores inflamatórios (TAPIA et al., 2012). Os mesmos pesquisadores relataram posteriormente que, ao aumentar-se a dose terapêutica de curcumina, foram revertidas alterações estruturais renais, proteinúria, hipertensão, fibrose intersticial, glomérulos afetados, atrofia tubular e expansão mesangial, além do próprio estresse oxidativo (TAPIA et al., 2013).

O intestino pode ser acometido por injúrias de $\mathrm{I} / \mathrm{R}$ devido à isquemia mesentérica aguda, transplante de intestino delgado, aneurisma da aorta abdominal, choque hemorrágico, traumático ou séptico ou queimaduras intensas, entre outros. As injúrias provocadas por $\mathrm{I} / \mathrm{R}$ intestinal são de cunho relevante, pois induzem danos a órgãos distantes, principalmente pulmões. Porém, já foram relatadas também injúrias em outros órgãos vitais como coração, fígado e rins; os principais causadores dessas lesões remotas foram as EROs e os leucócitos. Alguns estudos demonstraram que a curcumina atuou efetivamente na proteção tanto intestinal quanto dos demais órgãos acometidos. A suplementação com o composto por quinze dias teve efeito protetor na lesão pulmonar aguda induzida pela I/R intestinal, visualizada pela baixa marcação, comparada ao grupo sem tratamento, de anticorpos anti-COX2 por meio da técnica de imunoistoquímica (OKUDAN et al., 2013). Avaliou-se também, em outro estudo, os efeitos anti-inflamatórios e antioxidantes nos tecidos intestinal, pulmonar e cardíaco de ratos e foi verificado que níveis de MDA estiveram aumentados durante a $\mathrm{l} / \mathrm{R}$ e foram reduzidos com a 
administração da curcumina. A enzima SOD apresentou-se aumentada, o que comprovou o efeito contra o estresse oxidativo. O pré tratamento com curcumina evitou também a produção de citocinas pró-inflamatórias (EMBABY, 2014).

\section{CONSIDERAÇÕES FINAIS}

A cúrcuma (Curcuma Longa L.) é uma planta utilizada há séculos, com vários propósitos. Recentemente, seu uso tem se ampliado em escala mundial a cada comprovação científica de seus efeitos terapêuticos relatados desde a antiguidade. Sua importância econômica evidencia, ainda mais, a importância de seu uso tanto na culinária, como condimento, quanto na promoção da saúde humana e animal.

A estrutura molecular do principal componente da cúrcuma, a curcumina, apresenta sítios de reações antioxidantes altamente benéfico, perante transtornos de I/R, os quais apresentam como consequência produção em demasia de ERO. Nesses casos, a ação da curcumina pode ser direta, por meio de ligação química às moléculas reativas ou indireta, induzindo a expressão de enzimas antioxidantes.

O uso da molécula em modelos animais de experimentação tem viabilizado o entendimento dos mecanismos de ação da curcumina, não somente como antioxidante, mas como anti-inflamatória e antiapoptótica. Esses resultados podem ser extrapolados para seres humanos e animais, que se beneficiarão de uma fórmula terapêutica, com vários sítios de atuação nas diversas doenças.

O uso de compostos fenólicos em pacientes humanos e animais acometidos por neoplasias já é amplamente relatado na literatura. Por outro lado, seu emprego em animais acometidos por injúrias de $\mathrm{I} / \mathrm{R}$, como por exemplo a injúria renal aguda, pode ser um avanço considerável, haja vista que é uma manifestação frequente, na clínica de animais de companhia.

Para tanto necessita-se de aprimoramento científico no que tange a padronização da técnica de extração da curcumina, biodisponibilidade da molécula, dose efetiva, para sua utilização pura ou como precursora de fármacos semisintéticos.

\section{REFERÊNCIAS}

ANJOMSHOA, S., NAMAZIAN, M., NOORBALA, M. R. The Effect of Solvent on Tautomerism, Acidity and Radical Stability of Curcumin and Its Derivatives Based on Thermodynamic Quantities. Journal of Solution Chemistry. v. 45, p. 1021, 2016. https://doi.org/10.1007/s10953-016-0481-y

ALONSO, J. Tratado de Fitofármacos y Nutracéuticos. Argentina: Corpus. p. 373381, 2007.

BANIK, U., PARASURAMAN, S., ADHIKARY, A. K., ORTHMAN, N. H. Curcumin: the spicy modulator of breast carcinogenesis. Journal of Experimental \& Clinical Cancer Research, v. 36, n. 1, p. 98. 2017. Doi: 10.1186/s13046-017-0566-5

BRASIL. Ministério da Saúde; Portal da Saúde: Programa Nacional de Plantas Medicinais e Fitoterápicos. 1a ed. Brasília: Editora MS, 135, 2009. 
BIANCHI, M. D. L. P., ANTUNES, L. M. G. Radicais livres e os princípios antioxidantes da dieta. Revista de Nutrição, Campinas, v. 12, n. 2, p. 23, 1999. http://dx.doi.org/10.1590/S1415-52731999000200001

BRASIL. Saúde Brasil 2013: uma análise da situação de saúde e das doenças transmissíveis relacionadas à pobreza. 10a ed, Brasília, 2014.

BRASIL. Ministério da Saúde. Portal da Saúde: Curcuma longa L. (CURCUMA). Brasília: 2015. 163.

DANTAS, F. Identidade ao açafrão de Mara Rosa. Revista Safra, Goiânia, 20 de maio de 2016, Agricultura, pag 28. [Acesso 17 set 2018]. Disponível em http://revistasafra.com.br/identidade- ao-acafrao-de-mara-rosa/

EMBABY, A. S. Histological and immunohistochemical study on the possible protective effect of curcumin on intestinal ischemia/reperfusion-induced lung injury in albino rats. The Egyptian Journal of Histology. v. 37, n. 1, p. 16-23, 2014. Doi: 10.1097/01.EHX.0000444372.52068.e7

GAUI, E. N., OLIVEIRA, G. M., KLEIN, C. H. Mortalidade por Insuficiência Cardíaca e Doença Isquêmica do Coração no Brasil de 1996 a 2011. Arquivo Brasileiro de Cardiologia, v. 102, n. 6, p. 557-65, 2014.

GHOLAMPOUR, F., SADIDI, Z. Hepatorenal protection during renal ischemia by quercetin and remote ischemic preconditioning. Journal of Surgical Research. v. 231, p. 224-233, 2018 https://doi.org/10.1016/j.jss.2018.05.036

HAUSENLOY D. J., YELLON, D. M. Myocardial ischemia-reperfusion injury: a neglected therapeutic target. The Journal of Clinical Investigation. v. 23, n. 1, p. 92-100, 2013. Doi: 10.1172/JCI62874

JACOB, A., CHAVES, L., EADON, M.T., CHANG, A., QUIGG, R.J., ALEXANDER, J. $J$. Curcumin alleviates immune-complex mediated glomerulonephritis in factor $\mathrm{H}$ deficient mice. Immunology. v. 139, p. 328-37, 2013.

KALOGERIS, T., BAINES, C.P., KRENZ, M., KORTHUIS, R.J. Ischemia/Reperfusion. Comprehensive Physiology. v. 7, p. 113-70, 2016. Doi: 10.1002/cphy.c160006

KUMAR, V., ABBAS, A. K.; ASTER, J. C. Robbins patologia básica. 9. ed. Rio de Janeiro: Elsevier, 2013.

LI, W., SUWANWELA, N. C, PATUMRAJ, S. Curcumin prevents reperfusion injury following ischemic stroke in rats via inhibition of NF-kB, ICAM-1, MMP-9 and caspase-3 expression. Molecular Medicine Reports. v. 16, p. 4710-20, 2017. DOI: $10.3892 / \mathrm{mmr} .2017 .7205$ 
LIU, H., WANG, C., QIAO, Z., XU, Y. Protective effect of curcumin against myocardium injury in ischemia reperfusion rats. Pharmaceutical Biology. v. 55, n. 1, p. 1144-48, 2017. DOI: 10.1080/13880209.2016.1214741

LIU, Z., RAN, Y., HUANG, S., WEN, S., ZHANG, W., et al. Curcumin Protects against Ischemic Stroke by Titrating Microglia/Macrophage Polarization. Frontiers in Aging Neuroscience. vol. 9, p. 1-10, 2017. Doi: 10.3389/fnagi.2017.00233

MARCHI, J. P., TEDESCO, L., MELO, A. C., FRASSON, A.C., FRANÇA, V. F., SATO, SW, LOVATO ECW. Curcuma longa L., o açafrão da terra, e seus benefícios medicinais. Arquivos de Ciências da Saúde UNIPAR, Umuarama, v. 20, n. 3, p. 189-194, 2016.

MIROŃCZUK-CHODAKOWSKA, I., WITKOWSKA, A. M., ZUJKO, M. E. Endogenous non-enzymatic antioxidants in the human body. Advances in Medical Sciences. $V$. 63, n. 1, p. 68, 2018. https://doi.org/10.1016/j.advms.2017.05.005

MOZAFFARIAN, D., BENJAMIN, E. J., GO A. S., ARNETT, D. K., BLAHA, M.J. et al., Heart disease and stroke statistics-2016 update: a report from the American Heart Association, Circulation. v. 133, n. 4, p. e38-e360, 2016. DOI: 10.1161/CIR.0000000000000350

OKUDAN, N., BELVIRAN, M., GÖKBEL, H., ÖZ, M., KUMAK, A. Protective effects of curcumin supplementation on intestinal ischemia reperfusion injury. Phytomedicine. v. 20, p. 844-48, 2013. Doi: 10.1016/j.phymed.2013.03.022

OZAWA, H., IMAIZUMI, A., SUMI, Y., HASHIMOTO, T., KANAI, M., et al. Curcumin $\beta$-D-Glucuronide Plays an Important Role to Keep High Levels of Free-Form Curcumin in the Blood. Biological and Pharmaceutical Bulletin. 2017;40(9);151524. http://doi.org/10.1248/bpb.b17-00339

PANDEY, K. B., RIZVI, S. I. Plant polyphenols as dietary antioxidants in human health and disease. Oxid Med Cell Longev. v. 2, n. 5, p. 270-78, 2009.

PONNUCHAMY, B., KHALIL, R. A. Cellular mediators of renal vascular dysfunction in hypertension. American Journal of Physiology- Regulatory Integrative and Comparative Physiology. v. 296, n. 4, p. 1001-18, 2009. Doi: 10.1152/ajpregu.90960.2008

PRABHAKARAN, N. K. P. The Agronomy and Economy of Turmeric and Ginger: the Invaluable Medicinal Spice Crops. London:Elsevier, p. 17-20, 2013.

PRIYADARSINI, K. I. The Chemistry of Curcumin: From Extraction to Therapeutic Agent. Molecules. vol. 19, n. 12, p. 20091-112, 2014. Doi: http://www.mdpi.com/1420-3049/19/12/20091

SILVA FILHO, C. R. M., SOUZA, A. G., CONCEIÇÃO, M. M., SILVA, T. G., SILVA, T. M. S., RIBEIRO, A. P. L. Avaliação da bioatividade dos extratos de cúrcuma (Curcuma longa L., Zingiberaceae) em Artemia salina e Biomphalaria glabrata. 
Revista Brasileira de Farmacognosia, v. 19, n. 4, p. 919-23, 2009. http://dx.doi.org/10.1590/S0102-695X2009000600022

SILVA JUNIOR, O. C., CENTURION S., PACHECO, E. G., BRISOTTI, J. L., OLIVEIRA, A. F., SASSO, K. D. Aspectos básicos da lesão de isquemia e reperfusão e do pré-condicionamento isquêmico. Acta Cirúrgica Brasileira. v. 17, n. 13, p. 1519, 2002. http://dx.doi.org/10.1590/S0102-86502002000900020.

SHOJI, M., NAKAGAWA, K., WATANABE, A., TSUDUKI, T., YAMADA, T., et al. Comparison of the effects of curcumin and curcumin glucuronide in human hepatocellular carcinoma HepG2 cells. Food Chemistry. 2014;151;126-32. https://doi.org/10.1016/j.foodchem.2013.11.021

TAPIA, E., SOTO, V., ORTIZ-VEGA, K. M., ZARCO-MÁRQUEZ, G., MOLINA-JIJÓN E., et al. Curcumin induces Nrf2 nuclear translocation and prevents glomerular hypertension, hyperfiltration, oxidant stress, and the decrease in antioxidant enzymes in 5/6 nephrectomized rats. Oxidative Medicine and Cellular Longevity, p. 1-14, 2012. http://dx.doi.org/10.1155/2012/269039

TAPIA E, ZATARAIN-BARRÓN ZL, HERNÁNDEZ-PANDO R, ZARCO-MÁRQUEZ G, MOLINA-JIJÓN E, et al. Curcumin reverses glomerular hemodynamic alterations and oxidant stress in 5/6 nephrectomized rats. Phytomedicine. v. 20, n. 3-4, p. 359-66, 2013. Doi: 10.1016/j.phymed.2012.11.014

VERCESI, A. E, CASTILHO, R. F, KOWALTOWSKI, A. J, OLIVEIRA, H. C. F., SOUZA-PINTO, N. C., et al. Mitochondrial calcium transport and the redox nature of the calcium-induced membrane permeability transition. Free Radical Biology and Medicine. v. 129, p. 1-24, 2018. https://doi.org/10.1016/j.freeradbiomed.2018.08.034

XU, Y., HU, N., JIANG, W., YUAN, H.F., ZHENG, D.H. Curcumin-carrying nanoparticles prevent ischemia-reperfusion injury in human renal cells. Oncotarget. v. 7, n. 52, p. 87390-401, 2016. Doi: 10.18632/oncotarget.13626.

YANG, Y., DUAN, W., LIN, Y., YI, W., LIANG, Z., et al. Sirt1 activation by curcumin pretreatment attenuates mitochondrial oxidative damage induced by myocardial ischemia reperfusion injury. Free Radical Biology \&. Medicine. v. 65, p. 667-79, 2013. DOI: https://doi.org/10.1016/j.freeradbiomed.2013.07.007

ZUJKO, M. E., WITKOWSKA, A. M., WAŚKIEWICZ, A., SYGNOWSKA, E. Estimation of dietary intake and patterns of polyphenol consumption in Polish adult population. Advances in Medical Sciencies. v. 57, n. 2, p. 375-84, 2012. https://doi.org/10.2478/v10039-012-0026-6 